\title{
Discrete Symmetry Transformations of Third Order Ordinary Differential Equations and Applications
}

\author{
KHUDIJA BIBI ${ }^{1,2}$, TOOBA FEROZE ${ }^{1}$ \\ 1. Department of Mathematics, School of Natural Sciences \\ National University of Sciences and Technology \\ Islamabad, PAKISTAN
}

2. Department of Mathematics, Faculty of Basic and Applied Sciences

International Islamic University, Islamabad, PAKISTAN

\begin{abstract}
Third order ordinary differential equations have already been classified by the Lie algebra they admit. Invariant equations corresponding to these Lie algebras are also available in the literature [17]. In thils paper, list of all discrete symmetries corresponding to these invariant ordinary differential equations, areobtained.Someparticularexamplesaregiventoshowthesignificanceofthework.
\end{abstract}

Key-Words: - Third order invariants, Lie algebra, discrete symmetry transformations

Received: April 12, 2020. Revised: October 4, 2020. Accepted: October 14, 2020. Published: October 27, 2020.

\section{Introduction}

Group theoretic method introduced by Sophus Lie, is a useful technique to solve the differential equations in different branches of engineering, economics, natural and applied sciences. This method is based on group of transformations, called the Lie point symmetry groups of transformations of differential equations [1, 2, 3, 4. These groups of transformations depend upon continuous parameter(s) and are known as continuous groups. Lie, developed an systematic way to determine these symmetry groups associated with differential equations 2, 4, 5]. The symmetry group of a differential equation can be used to investigate the differential equation in various ways such as to solve or decrease the order of the given differential equation 1, 6, to generate new solutions from old ones 3, 6, to linearize a differential equation (if possible), [7, 8, 9] and to find conserved quantities [6] etc.

Apart from continuous transformations, there exist other type of symmetries which do not depend upon the parameter(s). However, they have great importance. Discrete symmetry transformations are one of them [1, 10. Following are some applications of these symmetries in the area of differential equations

- to improve the convergence of the numerical scheme of partial differential equations [11],

- to obtain the particular solutions of the nonlinear ordinary differential equations [12]
- to derive new exact solutions from the known solutions 13 and

- to determine the type of bifurcations in dynamical systems [14.

The technique developed by Hydon [1] gives all discrete point symmetries of the differential equations. It is based on the Lie algebra, $\ell$, of Lie point symmetry generators of a differential equation.

\subsection{Discrete Symmetries of Ordinary} Differential Equations

Ordinary differential equations (ODE) of the form

$$
\mathrm{u}^{(n)}=\mathbb{G}\left(\mathbf{z}, \mathbf{u}, \dot{\mathrm{u}}, \ldots, \mathbf{u}^{(n-1)}\right), \quad n \geq 2,
$$

always have a finite dimensional Lie algebra $\ell$ of point symmetry generators (if it exists) with basis

$$
K_{i}=\xi_{i}(\mathrm{z}, \mathrm{u}) \partial_{\mathrm{z}}+\eta_{i}(\mathrm{z}, \mathrm{u}) \partial_{\mathrm{u}}, i=1,2 \ldots N,
$$

where $N=\operatorname{dim}(\ell)$.

The method developed by Hydon categorizes conceivable automorphisms of $\ell$, factoring out those which are equivalent under the action of any symmetry in Lie group generated by $\ell$. The change in basis vectors, $\mathbf{X}_{i}$, is

$$
\mathbf{X}_{i}=b_{i}^{l} \mathbf{X}_{l} .
$$

As the structure constants do change under the trasformation, they follow the transformation law given below

$$
c_{l m}^{n} b_{i}^{l} b_{j}^{m}=c_{i j}^{k} b_{k}^{n}, \quad i, j, k, l, m, n=1, \cdots, r .
$$


These constraints provide a real-valued matrix $B=\left(b_{i}^{l}\right)$ that corresponds to the automorphism. Then it will be possible to attain the most general realization of the inequivalent automorphism as a point transformation. Lastly replacing these transformations into symmetry condition

$$
\hat{\mathrm{u}}^{(n)}=\mathbb{G}\left(\hat{z}, \hat{\mathrm{u}}, \ldots, \hat{\mathrm{u}}^{(n-1)}\right),
$$

one can get a complete list of discrete symmetry groups of the ODEs (2) [15]. Following example elaborates the technique:

Example

Consider the following third order ODE [16]

$$
\dddot{u}+u \ddot{u}-\dot{u}^{2}=0,
$$

which has the following two dimensional Lie algebra

$$
K_{1}=\partial_{\mathrm{z}}, K_{2}=\mathrm{z} \partial_{\mathrm{z}}+\mathrm{u} \partial_{\mathrm{u}} .
$$

This Lie algebra is non-abelian and its only nonzero structure constant is

$$
c_{12}^{1}=1 \text {. }
$$

The elements of the matrix $B$ satisfy the following system of nonlinear constraints

$$
c_{l m}^{n} b_{i}^{l} b_{j}^{m}=c_{i j}^{k} b_{k}^{n}, \quad 1 \leq i<j \leq N, \quad 1 \leq n \leq N .
$$

For $n=2$, the above constraints become

$$
c_{i j}^{k} b_{k}^{2}=0, \quad 1 \leq i<j \leq 2 .
$$

From above, we get only one value by setting $(i, j)=(1,2)$ which is

$$
b_{1}^{2}=0 .
$$

Similarly for $n=1$ in (6), we have

$$
b_{1}^{1}=b_{1}^{1} b_{2}^{2}-b_{1}^{2} b_{2}^{1} .
$$

As $b_{1}^{2}=0$, so above equation becomes

$$
b_{2}^{2}=1, b_{1}^{1} \neq 0 .
$$

Now the non-singular matrix $B$ becomes

$$
B=\left[\begin{array}{ll}
b_{1}^{1} & 0 \\
b_{2}^{1} & 1
\end{array}\right] .
$$

The matrices corresponding to the automorphisms generated by $K_{1}$ and $K_{2}$ given in (6) are

$$
A(1, \epsilon)=\left[\begin{array}{cc}
1 & 0 \\
-\epsilon & 1
\end{array}\right], A(2, \epsilon)=\left[\begin{array}{cc}
e^{\epsilon} & 0 \\
0 & 1
\end{array}\right] .
$$

For further simplification of $B$, premultiply it by $A\left(1, \frac{b_{2}^{1}}{b_{1}^{1}}\right)$ to replace $b_{2}^{1}$ by zero and premultiply $B$ by $A\left(2,-\ln \left|b_{1}^{1}\right|\right)$ to replace $b_{1}^{1}$ by $\alpha$, so now

$$
B=\left[\begin{array}{cc}
\alpha & 0 \\
0 & 1
\end{array}\right], \quad \alpha= \pm 1
$$

We have to solve the determining equations

$$
\left[\begin{array}{cc}
\hat{z}_{z} & \hat{u}_{z} \\
z \hat{z}_{z}+u \hat{z}_{u} & z \hat{u}_{z}+u \hat{u}_{u}
\end{array}\right]=B\left[\begin{array}{cc}
1 & 0 \\
\hat{z} & \hat{u}
\end{array}\right]=\left[\begin{array}{cc}
\alpha & 0 \\
\hat{z} & \hat{u}
\end{array}\right] .
$$

The general solution of the determining equations is

$$
\hat{z}=\alpha z, \hat{u}=c u,
$$

where $c$ is the constant of integration.

From (7),

$$
\begin{gathered}
\dot{\hat{u}}=\frac{c}{\alpha} \dot{u}, \\
\ddot{\hat{u}}=\frac{c}{\alpha^{2}} \ddot{u}, \alpha^{2}=1, \\
\cdots \\
\hat{u}=\frac{c}{\alpha} \dddot{u} .
\end{gathered}
$$

Putting above results into the symmetry condition (3), we find that either

$$
(\hat{z}, \hat{u})=(z, u),
$$

or

$$
(\hat{z}, \hat{u})=(-z,-u)
$$

Thus the group of discrete symmetries of (4) is generated by

$$
v_{1}:(z, u) \mapsto(-z,-u),
$$

which is isomorphic to $\mathbb{Z}_{2}$.

\section{Third Order Invariant Equations with Discrete Symmetry Transformations}

In this section, a complete list of the discrete symmetry transformations for third order ordinary differential equations is provided. Considering the Lie algebra and third order invariant differential equations, the discrete symmetry groups are obtained. It is known that third order ODEs may admit one, two, three, four, six and seven dimensional algebras [17]. Here each class has been separately considered to get the discrete symmetries and the results are given in the form of tables. In these tables $\mathrm{f}$ is an arbitrary function and $C$ is an arbitrary constant. 


\subsection{One Dimensional Algebra}

The only possible one-dimensional Lie algebra [17], corresponding invariant ODE and the respective discrete symmetry group are given in Table 1.

\subsection{Two Dimensional Algebra}

In Table 2, two dimensional algebras, invariant equations [17, 18, 19] and the obtained discrete symmetries are presented. where $c$ is the constant of integration.

\subsection{Three dimensional Algebra}

Table 3 displays the discrete symmetries of those third order invariant equations which remain invariant under three dimensional algebra.

\subsection{Four Dimensional Algebra}

Table 4 shows the discrete symmetries corresponding to third order ODEs, admitting four dimensional algebra.

\subsection{Six Dimensional Algebra}

There are two distinct six dimensional algebras that third order differential equations may admit [17. Discrete symmetries corresponding in both cases are given in Table 5 .

\subsection{Seven Dimensional Algebra}

Following table shows the discrete symmetries for the maximal dimensional Lie algebra that a third order ordinary differential equation can obtain.

\section{Examples}

1. Consider the following ODE

$$
\dddot{\mathrm{u}}=\frac{\ddot{\mathrm{u}}^{2}}{\dot{\mathrm{u}}}+\frac{\ddot{\mathrm{u}}}{\mathrm{z}}+\frac{\dot{\mathrm{u}}}{\mathrm{z}},
$$

which is invariant under the following two dimensional Lie algebra $\partial_{\mathbf{u}}, \mathbf{u} \partial_{\mathbf{u}}$. From the Table 2 , without calculations one can find that its discrete symmetry is $(\hat{z}, \hat{u})=(z,-u)$.

2. The third-order Riccati Hierarchy differential equation 20.

$$
\dddot{u}+4 u \ddot{u}+3 \dot{u}^{2}+6 \dot{u} u^{2}+u^{4}=0,
$$

is invariant under the following Lie point symmetry generator

$$
\mathrm{K}_{1}=\partial_{\mathrm{z}}, \quad \mathrm{K}_{2}=\mathrm{z} \partial_{\mathrm{z}}-\mathrm{u} \partial_{\mathrm{u}},
$$

and its discrete symmetry can be found from Table 2, which is

$$
(\hat{z}, \hat{u})=(-z,-u) .
$$

3. Consider another ODE, given in [21]

$$
\dddot{\mathrm{u}}=\ddot{\mathrm{u}}-(\ddot{\mathrm{u}})^{2} \text {. }
$$

which is invariant under the following three dimensional Lie algebra

$$
\mathrm{K}_{1}=\partial_{\mathrm{z}}, \mathrm{K}_{2}=\partial_{\mathrm{u}}, \mathrm{K}_{3}=\mathrm{z} \partial_{\mathrm{u}}
$$

By using Table 3, we have found that discrete symmetry of above ODE is

$$
(\hat{z}, \hat{u})=\left(-z, \frac{1}{2} z^{2}-u\right) .
$$

3.1 Solutions of some ODEs using discrete symmetry groups

1. Consider the Blasius equation

$$
\dddot{u}+\frac{1}{2} u \ddot{u}=0,
$$

which is invariant under two dimensional Lie algebra $\partial_{z}, z \partial_{z}-u \partial_{u}$ and its only discrete symmetry is $(-z, u)$. Then $u=f(-z)$ or $u=-z$ is the solution of (10).

2. Consider the following ODE

$$
\dddot{u}=\frac{3}{2} \frac{\ddot{u}^{2}}{\dot{\mathrm{u}}},
$$

then from Table 5 , it has found that $\left(\frac{1}{\mathrm{z}}, \mathrm{u}\right)$ and $(-\mathbf{z}, \mathbf{u})$ are discrete symmetries of above 
Table 1:

\begin{tabular}{|c|c|c|c|}
\hline & Algebra & Invariant Equation & Discrete Transformations \\
\hline$L_{1}$ & $\partial_{\mathbf{z}}$ & $\dddot{\mathrm{u}}=\mathrm{f}(\mathrm{u}, \dot{\mathrm{u}}, \ddot{\mathrm{u}})$ & $v_{1}:(\mathrm{z}, \mathrm{u}) \mapsto\left(b_{1}^{1} \mathrm{z}, \mathrm{u}\right), \quad b_{1}^{1} \neq 0$ \\
\hline
\end{tabular}

Table 2:

\begin{tabular}{|c|c|c|c|}
\hline & Algebra & $\begin{array}{l}\text { Invariant } \\
\text { Equation }\end{array}$ & Discrete Transformations \\
\hline$L_{2,1}^{I}$ & $\partial_{\mathbf{z}}, \partial_{\mathrm{u}}$ & $\dddot{\mathrm{u}} \dddot{\mathrm{u}}=\mathrm{f}(\dot{\mathrm{u}}, \ddot{\mathrm{u}})$ & $v_{1}:(\mathbf{z}, \mathbf{u}) \mapsto\left(b_{1}^{1} \mathbf{z}+b_{2}^{1} \mathrm{u}, b_{1}^{2} \mathbf{z}+b_{2}^{2} \mathbf{u}\right), b_{1}^{1} b_{2}^{2}-b_{1}^{2} b_{2}^{1} \neq 0$ \\
\hline$L_{2,1}^{I I}$ & $\partial_{\mathrm{u}}, \mathrm{z} \partial_{\mathrm{u}}$ & $\dddot{\mathrm{u}}=\mathrm{f}(\mathrm{z}, \mathrm{ü})$ & $v_{1}:(\mathrm{z}, \mathrm{u}) \mapsto\left(\frac{b_{2}^{1}-b_{1}^{1} \mathrm{z}}{b_{1}^{2} \mathrm{z}-b_{2}^{2}}, \frac{b_{1}^{2} b_{2}^{1}-b_{1}^{1} b_{2}^{2}}{b_{1}^{2} \mathrm{z}-b_{2}^{2}} \mathrm{u}\right), b_{1}^{1} b_{2}^{2}-b_{1}^{2} b_{2}^{1} \neq 0$ \\
\hline$L_{2,2}^{I}$ & $\overline{\partial_{\mathrm{u}}, \mathrm{z} \partial_{\mathrm{z}}+\mathrm{u} \partial_{\mathrm{u}}}$ & $\dddot{u}=\ddot{u}^{2} \mathrm{f}(\dot{\mathrm{u}}, \mathrm{zü})$ & $v_{1}:(\mathrm{z}, \mathrm{u}) \mapsto(c \mathrm{z}, \alpha \mathrm{u}+\mathrm{zF}(\mathrm{u})), \alpha= \pm 1, F(0)=0$ \\
\hline$L_{2,2}^{I I}$ & $\partial_{\mathrm{u}}, \mathrm{u} \partial_{\mathrm{u}}$ & $\dddot{u}=\dot{u} f(z, \ddot{u} / \dot{u})$ & $v_{1}:(\mathrm{z}, \mathrm{u}) \mapsto(\mathrm{z},-\mathrm{u})$ \\
\hline & $\partial_{\mathrm{z}}, \mathrm{z} \partial_{\mathrm{z}}-\mathrm{u} \partial_{\mathrm{u}}$ & $\dddot{\mathrm{u}}=\mathrm{f}(\mathrm{u}, \dot{\mathrm{u}}, \ddot{\mathrm{u}})$ & $v_{1}:(\mathrm{z}, \mathrm{u}) \mapsto(\alpha \mathrm{z}, c \mathrm{u}), \alpha= \pm 1$ \\
\hline
\end{tabular}

ODE. The solutions of ODE (11) that are invariant under these transformations will be of the form

$$
\mathrm{u}=\mathrm{f}_{1}\left(\frac{1}{\mathrm{z}}\right) \text { and } \mathrm{u}=\mathrm{f}_{2}(-\mathrm{z}) .
$$

By simply taking $\mathrm{f}_{1}\left(\frac{1}{\mathrm{z}}\right)=\frac{1}{\mathrm{z}}$ and $\mathrm{f}_{2}(-\mathrm{z})$ $=-\mathrm{z}$, then $\mathrm{u}=\frac{1}{\mathrm{z}}$ and $\mathrm{u}=-\mathrm{z}$ are the solutions of (11), which have been obtained by using its discrete symmetry transformations.

3. The discrete symmetry group of the ODE (8) is given in (9). Thus the solution of (8) invariant under this discrete transformation is of the form

$$
\frac{1}{2} \mathrm{z}^{2}-\mathrm{u}=\mathrm{f}(-\mathrm{z})
$$

Thus it can be easily verify that

$$
\mathrm{u}=\frac{1}{2} \mathrm{z}^{2}+\mathrm{z}
$$

is the solution of ODE (8).

These examples illustrate that discrete symmetry transformations can be helpful to generate some new solutions of the ordinary differential equations.

\section{Conclusion}

In this article, we have obtained discrete symmetry transformations of third order ordinary differential equations by using their Lie algebras. During the computations, it was observed that discrete symmetries of ordinary differential equations can also be calculated more easily with the help of its subalgebras rather than by considering the whole algebra. For example, an ODE invariant under the six dimensional algebra given in Table 5(a), comprises all those five discrete symmetries which have been found by its different subalgebras (given in previous Tables) like one dimensional, two dimensional, three dimensional and four dimensional subalgebras. At the end of the article, some particular cases are discussed to generate new solutions that are invariant under discrete symmetry transformations.

\section{References:}

[1] P.E. Hydon, Symmetry Methods for Differential Equations: A Beginner's Guide, Cambridge university Press, Cambridge, (2000).

[2] H. Stephani, Differential equations: Their solutions using symmetries, Cambridge university Press, Cambridge, (1989).

[3] G.W. Bluman and S. Kumei, Symmetries and Differential Equations, Springer-Verlag, New York, (1989).

[4] N.H. Ibragimov, Elementary Lie Group Analysis and Ordinary Differential Equations, John wiley \& Sons, Chichester, UK, (1999).

[5] P.J. Olver, Equivalnce, invariants and symmetry, Cambridge university press, Cambridge.

[6] P.J. Olver, Applications of Lie Groups to Differential Equations, Springer-Verlag, New York, (1986). 
[7] G.W. Bluman and S. Kumei, Symmetry based algorithms to relate partial differential equations: I. Local symmetries, Eur. J. Appl. Math, 1, (1990): 189-216.

[8] S. Kumei and G.W. Bluman, when nonlinear differential equations are equivalent to linear differential equations, SIAM J. Appl. Math, 42, (1982): 1157-1173.

[9] F.M. Mahomed and P. G.L. Leach, The Lie algebra $\mathrm{sl}(3, \mathrm{R})$ and linearization, Quaestiones Mathematicae, 12, (1989): 121-139.

[10] P.E. Hydon, How to use Lie symmetries to find discrete symmetries, MARS Publishers, Trondheim, (1999): 141-147.

[11] K. Bibi, T. Feroze, Discrete Symmetry Group Approach for Numerical Solution of the Heat Equation, Symmetry, (2020): 12, 180 .

[12] K. Bibi, Particular Solutions of Ordinary Differential Equations Using Discrete Symmetry Groups, Symmetry, (2020): 12, 359.

[13] H. Yang, Y. Shi, B. Yin, H. Dong, Discrete Symmetries Analysis and Exact Solutions of the Inviscid Burgers Equation, Discrete Dynamics in Nature and Society, 56, (2012): 115.

[14] M. Golubitsky, I. Stewart and D.G. Schaeffer, Singularities and Groups in Bifurcation Theory, Vol.II, Springer, New York, (1988).

[15] P.E. Hydon, Discrete point symmetries of ordinary differential equations, The Royal Society of London. Proceedings A, 454, (1998): 1961-1972.

[16] D.J. Arrigo, Symmetry analysis of differential equations: An introduction, John Wiley Sons, (2015).

[17] N.H. Ibragimov, CRC Handbook of Lie Group Analysis of Differential Equations, Vol.3, CRC Press, Boca Raton, (1996).

[18] R. Naz, F.M. Mahomed and D.P. Mason, Symmetry Solutions of a Third-Order Ordinary Differential Equation which Arises from
Prandtl Boundary Layer Equations, Journal of Nonlinear Mathematical Physics, 15, (1998): 179-191.

[19] N.H. Ibragimov and M.C. Nucci, Integration of third-order ordinary differential equations by Lie's method: Equations admitting three-dimensional lie algebras, Lie Groups and Their Applications, 1, (1994): 49-64.

[20] W.F. Ames, Nonlinear ordinary differential equations in transport processes, Academic Press, New York, (1968).

[21] P.E. Hydon, Discrete Symmetries of Differential Equations, Cambridge university Press, Cambridge, (2000).

Contribution of individual authors to the creation of a scientific article (ghostwriting policy)

Author Contributions:

Supervised by Tooba Feroze.

Calculations and write up of the article is done by Khudija Bibi.

Follow: www.wseas.org/multimedia/contributorrole-instruction.pdf

Sources of funding for research presented in a scientific article or scientific article itself

National University of Sciences and Technology Islamabad, Pakistan

\section{Creative Commons Attribution License 4.0 (Attribution 4.0 International, CC BY 4.0)}

This article is published under the terms of the Creative Commons Attribution License 4.0 https://creativecommons.org/licenses/by/4.0/deed.en_US 
Table 3:

\begin{tabular}{|c|c|c|c|}
\hline & Algebra & $\begin{array}{l}\text { Invariant } \\
\text { Equation }\end{array}$ & Discrete Transformations \\
\hline$L_{3,1}$ & $\partial_{\mathrm{u}}, \mathrm{z} \partial_{\mathrm{u}}, h(\mathrm{z}) \partial_{\mathrm{u}}$ & $\dddot{\mathrm{u}}=\frac{\dddot{h}(\mathrm{z})}{\ddot{h}(\mathrm{z})} \ddot{\mathrm{u}}+\mathrm{f}(\mathrm{z})$ & $\begin{array}{c}v_{1}:(\mathrm{z}, \mathrm{u}) \mapsto\left(\mathrm{z}, b_{1}^{1} \mathrm{u}\right. \\
b_{1}^{1}=b_{2}^{2}=b_{3}^{3} \neq 0\end{array}$ \\
\hline$L_{3,2}$ & $\partial_{\mathrm{u}}, \partial_{\mathrm{z}}, \mathrm{z} \partial_{\mathrm{u}}$ & $\dddot{\mathrm{u}}=\mathrm{f}(\ddot{\mathrm{u}})$ & $\begin{aligned} v_{1}:(\mathrm{z}, \mathrm{u}) \mapsto & \left(b_{1}^{1} \mathrm{z}, \frac{1}{2} b_{1}^{1} b_{1}^{2} \mathrm{z}^{2}+b_{1}^{1} b_{2}^{2} \mathrm{u}\right), \\
& b_{1}^{1} b_{2}^{2} \neq 0 .\end{aligned}$ \\
\hline$L_{3,3}^{I}$ & $\begin{array}{c}\partial_{\mathrm{u}}, \partial_{\mathbf{z}} \\
\mathrm{z} \partial_{z}+(\mathrm{z}+\mathrm{u}) \partial_{\mathrm{u}}\end{array}$ & $\dddot{\mathrm{u}}=\ddot{\mathrm{u}}^{2} \mathrm{f}(\ddot{\mathrm{u}} \exp (\dot{\mathrm{u}}))$ & $\begin{array}{c}v_{1}:(\mathrm{z}, \mathrm{u}) \mapsto\left(b_{2}^{2} \mathrm{z}, b_{2}^{1} \mathrm{z}+b_{1}^{1} \mathrm{u}\right) \\
b_{1}^{1} b_{2}^{2} \neq 0\end{array}$ \\
\hline$L_{3,3}^{I I}$ & $\partial_{\mathrm{u}}, \mathrm{z} \partial_{\mathrm{u}}, \partial_{\mathrm{z}}+\mathrm{u} \partial_{\mathrm{u}}$ & $\dddot{\mathrm{u}}=\ddot{\mathrm{u}} \mathrm{f}\left(\exp \left(\frac{\mathrm{z}}{\ddot{\mathrm{u}}}\right)\right)$ & $\begin{array}{c}v_{1}:(\mathbf{z}, \mathbf{u}) \mapsto\left(\mathbf{z}, b_{1}^{1} \mathbf{u}+\exp (\mathbf{z}) F(\mathbf{u})\right) \\
b_{1}^{1}=b_{2}^{2} \neq 0, F(0)=0 .\end{array}$ \\
\hline$L_{3,4}^{I}$ & $\partial_{\mathrm{z}}, \partial_{\mathrm{u}}, \mathrm{z} \partial_{\mathrm{z}}$ & $\dddot{\mathrm{u}}=\ddot{\mathrm{u}}^{\frac{3}{2}} \mathrm{f}\left(\ddot{\mathrm{u}} \dot{\mathrm{u}}^{-2}\right)$ & $\begin{array}{c}v_{1}:(\mathrm{z}, \mathrm{u}) \mapsto\left(\alpha \mathrm{z}, b_{2}^{2} \mathrm{u}\right) \\
b_{2}^{2} \neq 0, \alpha= \pm 1\end{array}$ \\
\hline$L_{3,4}^{I I}$ & $\partial_{\mathrm{u}}, \mathrm{z} \partial_{\mathrm{u}}, \mathrm{z} \partial_{\mathrm{z}}+\mathrm{u} \partial_{\mathrm{u}}$ & $\dddot{\mathrm{u}}=\ddot{\mathrm{u}}^{2} \mathrm{f}(\mathrm{z} \ddot{\mathrm{u}})$ & $\begin{array}{c}v_{1}:(\mathrm{z}, \mathrm{u}) \mapsto\left(\frac{\alpha}{b_{2}^{2}} \mathrm{z}, \alpha \mathrm{u}+\left(\frac{\alpha b_{3}^{2}}{b_{2}^{2}}\right) \mathrm{z} \ln \mathrm{z}+\right. \\
\mathrm{z} F(\mathrm{u})), b_{2}^{2} \neq 0, \alpha= \pm 1, \\
F(0)=0 .\end{array}$ \\
\hline$L_{3,5}^{I}$ & $\partial_{\mathrm{z}}, \partial_{\mathrm{u}}, \mathrm{z} \partial_{\mathrm{z}}+\mathrm{u} \partial_{\mathrm{u}}$ & $\dddot{\mathrm{u}}=\ddot{\mathrm{u}}^{2} \mathrm{f}(\dot{\mathrm{u}})$ & $\begin{aligned} v_{1}:(\mathbf{z}, \mathbf{u}) \mapsto & \left(b_{1}^{1} \mathbf{z}+b_{2}^{1} \mathbf{u}, b_{1}^{2} \mathbf{z}+b_{2}^{2} \mathbf{u}\right) \\
& b_{1}^{1} b_{2}^{2} \neq 0 .\end{aligned}$ \\
\hline$L_{3,5}^{I I}$ & $\partial_{\mathrm{u}}, \mathrm{z} \partial_{\mathrm{u}}, \mathrm{u} \partial_{\mathrm{u}}$ & $\dddot{\mathrm{u}}=\ddot{\mathrm{u}} \mathrm{f}(\mathrm{z})$ & $\begin{array}{c}v_{1}:(\mathbf{z}, \mathbf{u}) \mapsto\left(\mathbf{z}, b_{1}^{1} \mathbf{u}\right) \\
b_{1}^{1}=b_{2}^{2} \neq 0\end{array}$ \\
\hline$L_{3,6}^{I}$ & $\begin{array}{c}\partial_{\mathrm{z}}, \partial_{\mathrm{u}} \\
\mathrm{z} \partial_{\mathrm{z}}+a \mathrm{u} \partial_{\mathrm{u}} \\
a \neq 0,1,2\end{array}$ & $\dddot{\mathrm{u}}=\ddot{\mathrm{u}}^{\frac{a-3}{a-2}} \mathrm{f}\left(\ddot{\mathrm{u}}^{(1-a)} \dot{\mathrm{u}}^{(a-2)}\right)$ & $\begin{array}{c}v_{1}:(\mathrm{z}, \mathrm{u}) \mapsto\left(b_{1}^{1} \mathrm{z}, b_{2}^{2} \mathrm{u}\right) \\
b_{1}^{1} b_{2}^{2} \neq 0\end{array}$ \\
\hline$L_{3,6}^{I}$ & $\begin{array}{c}\partial_{\mathrm{z}}, \partial_{\mathrm{u}} \\
\mathrm{z} \partial_{\mathrm{z}}+2 \mathrm{u} \partial_{\mathrm{u}}, a=2\end{array}$ & $\dddot{\mathrm{u}}=\dot{\mathrm{u}}^{-1} \mathrm{f}(\ddot{\mathrm{u}})$ & $\begin{array}{c}v_{1}:(\mathrm{z}, \mathrm{u}) \mapsto\left(b_{1}^{1} \mathrm{z}, b_{2}^{2} \mathrm{u}\right) \\
b_{1}^{1} b_{2}^{2} \neq 0\end{array}$ \\
\hline$L_{3,6}^{I I}$ & $\begin{array}{c}\partial_{\mathrm{u}}, z \partial_{\mathrm{u}} \\
(1-a) \mathrm{z} \partial_{\mathrm{z}}+\mathrm{u} \partial_{\mathrm{u}} \\
a \neq 0,1, \frac{1}{2}\end{array}$ & $\dddot{\mathrm{u}}=\ddot{\mathrm{u}}^{\frac{2-3 a}{1-2 a}} \mathrm{f}\left(\mathrm{z}^{(1-2 a)} \ddot{\mathrm{u}}^{(1-a)}\right)$ & $\begin{array}{c}v_{1}:(\mathrm{z}, \mathrm{u}) \mapsto\left(\frac{b_{1}^{1}}{b_{2}^{2}} \mathrm{z}, b_{1}^{1} \mathrm{u}+\right. \\
\left.\mathrm{z}^{\frac{1}{1-a}} F(\mathrm{u})\right), \quad b_{1}^{1} b_{2}^{2} \neq 0 \\
F(0)=0\end{array}$ \\
\hline$L_{3,6}^{I I}$ & $\begin{array}{c}\partial_{\mathrm{u}}, z \partial_{\mathrm{u}} \\
\frac{1}{2} \mathrm{z} \partial_{\mathrm{z}}+\mathrm{u} \partial_{\mathrm{u}}, a=\frac{1}{2}\end{array}$ & $\dddot{\mathrm{u}}=\mathrm{z}^{-1} \mathrm{f}(\ddot{\mathrm{u}})$ & $\begin{array}{c}v_{1}:(\mathrm{z}, \mathrm{u}) \mapsto\left(\frac{b_{1}^{1}}{b_{2}^{2}} \mathrm{z}, b_{1}^{1} \mathrm{u}+\right. \\
\left.\mathrm{z}^{2} F(\mathrm{u})\right), \quad b_{1}^{1} b_{2}^{2} \neq 0 \\
F(0)=0\end{array}$ \\
\hline$L_{3,7}^{I}$ & $\begin{array}{l}\partial_{\mathrm{z}}, \partial_{\mathrm{u}},(b \mathrm{z}+\mathrm{u}) \partial_{\mathrm{z}} \\
\quad+(b u-z) \partial_{\mathrm{u}}\end{array}$ & $\begin{array}{c}\dddot{\mathrm{u}}=\frac{\ddot{\mathrm{u}}^{2}}{1+\dot{\mathrm{u}}^{2}}(3 \dot{\mathrm{u}}+ \\
\left.\mathrm{f}\left(\ddot{\mathrm{u}}\left(1+\dot{\mathrm{u}}^{2}\right)^{\frac{-3}{2}} \exp (-b \arctan \dot{\mathrm{u}})\right)\right)\end{array}$ & $\begin{aligned} v_{1}:(\mathrm{z}, \mathrm{u}) & \mapsto(-\mathrm{z},-\mathrm{u}) \\
v_{2}:(\mathrm{z}, \mathrm{u}) & \mapsto\left(b_{1}^{1} \mathrm{z}, b_{2}^{2} \mathrm{u}\right) \\
b_{1}^{1} b_{2}^{2} & \neq 0\end{aligned}$ \\
\hline$L_{3,7}^{I I}$ & $\begin{array}{l}\partial_{\mathrm{u}}, z \partial_{\mathrm{u}},\left(1+z^{2}\right) \partial_{\mathrm{z}} \\
\quad+(z+b) u \partial_{\mathrm{u}}\end{array}$ & $\begin{array}{c}\dddot{\mathrm{u}}=\frac{\mathrm{u}}{1+\mathrm{z}^{2}}(3 \mathrm{z}+ \\
\left.\mathrm{f}\left(\ddot{\mathrm{u}}\left(1+\mathrm{z}^{2}\right)^{\frac{3}{2}} \exp (-b \arctan \mathrm{z})\right)\right)\end{array}$ & $\begin{array}{c}v_{1}:(\mathrm{z}, \mathrm{u}) \mapsto\left(\mathrm{z}, b_{2}^{2} \mathrm{u}\right) \\
b_{1}^{1} b_{2}^{2} \neq 0, b_{1}^{1}=b_{2}^{2}\end{array}$ \\
\hline$L_{3,8}^{I}$ & $\begin{array}{c}\partial_{\mathrm{u}}, \mathrm{z} \partial_{\mathrm{z}}+\mathrm{u} \partial_{\mathrm{u}} \\
2 \mathrm{zu} \partial_{\mathrm{z}}+\mathrm{u}^{2} \partial_{\mathrm{u}}\end{array}$ & $\begin{array}{l}\dddot{u}=3=3 \ddot{\ddot{u}}^{2}+ \\
\frac{\dot{u}^{4}}{z^{2}} \mathrm{f}\left(\frac{2 z \ddot{u}+\dot{u}}{\dot{u}^{3}}\right)\end{array}$ & $\begin{aligned} v_{1}:(\mathrm{z}, \mathrm{u}) & \mapsto\left(c \mathrm{z}, b_{1}^{1} \mathrm{u}\right) \\
b_{1}^{1} & \neq 0 . \\
v_{2}:(\mathrm{z}, \mathrm{u}) & \mapsto(-\mathrm{z},-\mathrm{u})\end{aligned}$ \\
\hline$L_{3,8}^{I I}$ & $\begin{array}{c}\partial_{\mathrm{u}}, \mathrm{z} \partial_{\mathrm{z}}+\mathrm{u} \partial_{\mathrm{u}} \\
2 \mathrm{zu} \partial_{\mathrm{z}}+\left(\mathrm{u}^{2}-\mathrm{z}^{2}\right) \partial_{\mathrm{u}}\end{array}$ & $\begin{array}{c}\dddot{u}=\frac{3 \dot{u} \ddot{u}^{2}}{1+\dot{u}^{2}}+ \\
\frac{\left(1+\dot{u}^{2}\right)^{2}}{z^{2}} \mathrm{f}\left(\frac{z \ddot{u}-\dot{u}-\dot{u}^{3}}{\left(1+\dot{u}^{2}\right)^{\frac{3}{2}}}\right)\end{array}$ & $\begin{array}{c}v_{1}:(\mathrm{z}, \mathrm{u}) \mapsto(\mathrm{z}, \alpha \mathrm{u}) \\
\alpha= \pm 1\end{array}$ \\
\hline$L_{3,8}^{I I I}$ & $\begin{array}{c}\partial_{\mathrm{u}}, \mathrm{z} \partial_{\mathrm{z}}+\mathrm{u} \partial_{\mathrm{u}} \\
2 \mathrm{zu} \partial_{\mathrm{z}}+\left(\mathrm{u}^{2}+\mathrm{z}^{2}\right) \partial_{\mathrm{u}}\end{array}$ & 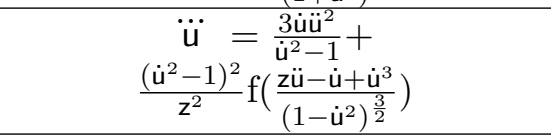 & $\begin{aligned} v_{1}:(\mathrm{z}, \mathrm{u}) & \mapsto(\mathrm{z},-\mathrm{u}) \\
v_{2}:(\mathrm{z}, \mathrm{u}) & \mapsto(-\mathrm{z}, \mathrm{u}) \\
v_{3}:(\mathrm{z}, \mathrm{u}) & \mapsto(-\mathrm{z},-\mathrm{u})\end{aligned}$ \\
\hline$L_{3,8}^{I V}$ & $\partial_{\mathrm{u}}, \mathrm{u} \partial_{\mathrm{u}}, \mathrm{u}^{2} \partial_{\mathrm{u}}$ & $\dddot{\mathrm{u}}=\frac{3}{2} \frac{\mathrm{\ddot { }}^{2}}{\dot{\mathrm{u}}}+\dot{\mathrm{u} f}(\mathrm{z})$ & $\begin{aligned} v_{1}:(\mathrm{z}, \mathrm{u}) & \mapsto(\mathrm{z},-\mathrm{u}), \\
v_{2}:(\mathrm{z}, \mathrm{u}) & \mapsto\left(\mathrm{z},-\frac{1}{\mathrm{u}}\right) \\
v_{2}:(\mathrm{z}, \mathrm{u}) & \mapsto\left(\mathrm{z}, \frac{1}{\mathrm{u}}\right) .\end{aligned}$ \\
\hline$L_{3,9}$ & $\begin{array}{l}\left(1+\mathrm{z}^{2}\right) \partial_{\mathrm{z}}+\mathrm{zu} \partial_{\mathrm{u}} \\
\mathrm{zu} \partial_{\mathrm{z}}+\left(1+\mathrm{u}^{2}\right) \partial_{\mathrm{u}} \\
\mathrm{u} \partial_{\mathrm{z}}-\mathrm{z} \partial_{\mathrm{u}}\end{array}$ & $\begin{array}{c}\dddot{\mathrm{u}}=\frac{-3 \mathrm{z} \ddot{\mathrm{u}}}{1+\mathrm{z}^{2}}+\frac{3 \ddot{\mathrm{u}}\left[\dot{\mathrm{u}}\left(1+\mathrm{z}^{2}\right)-\mathrm{zu}\right]}{1+\mathrm{z}^{2}} \\
{\left[\frac{\ddot{\mathrm{u}}\left(1+\mathrm{z}^{2}\right)}{1+\dot{\mathrm{u}}^{2}+(\mathrm{u}-\mathrm{z} \dot{\mathrm{u}})^{2}}-\frac{\mathrm{u}}{1+\mathrm{z}^{2}+\mathrm{u}^{2}}\right]} \\
+\frac{\left[1+\dot{\mathrm{u}}^{2}+(\mathrm{u}-\mathrm{z} \dot{\mathrm{u}})^{2}\right]^{2}}{\left(1+\mathrm{z}^{2}+\mathrm{u}^{2}\right)^{\frac{5}{2}}} \\
f\left(\ddot{\mathrm{u}}\left(\frac{1+\dot{\mathrm{u}}^{2}+(\mathbf{u}-\mathrm{u} \dot{u})^{2}}{1+\mathrm{z}^{2}+\mathrm{u}^{2}}\right)^{\frac{-3}{2}}\right)\end{array}$ & $\begin{array}{c}v_{1}:(\mathrm{z}, \mathrm{u}) \mapsto\left(b_{1}^{1} \mathrm{z}, b_{2}^{2} \mathrm{u}\right) \\
b_{1}^{1} b_{2}^{2} \neq 0 .\end{array}$ \\
\hline
\end{tabular}


Table 4:

\begin{tabular}{|c|c|c|c|}
\hline & Algebra & Invariant Equation & Discrete Transformations \\
\hline$L_{4,1}$ & $\partial_{\mathrm{u}}, \mathrm{z} \partial_{\mathrm{u}}, h(\mathrm{z}) \partial_{\mathrm{u}}, \mathrm{u} \partial_{\mathrm{u}}$ & $\dddot{\mathrm{u}}=\left(\frac{\dddot{h}(\mathrm{z})}{\ddot{h}(\mathrm{z})}\right) \ddot{\mathrm{u}}, \dddot{h} \neq 0$ & $\begin{array}{c}v_{1}:(\mathbf{z}, \mathbf{u}) \mapsto\left(\mathbf{z}, b_{1}^{1} \mathbf{u}\right. \\
b_{1}^{1}=b_{2}^{2}=b_{3}^{3} \neq 0\end{array}$ \\
\hline$L_{4,2}$ & $\partial_{\mathrm{z}}, \mathrm{z} \partial_{\mathrm{z}}, \partial_{\mathrm{u}}, \mathrm{u} \partial_{\mathrm{u}}$ & $\dddot{\mathrm{u}}=C\left(\frac{\ddot{\mathrm{u}}^{2}}{\dot{\mathrm{u}}}\right)$ & $v_{1}:(\mathrm{z}, \mathrm{u}) \mapsto(-\mathrm{z},-\mathrm{u})$ \\
\hline$L_{4,3}$ & $\begin{array}{c}\partial_{\mathrm{z}}, \partial_{\mathrm{u}}, \mathrm{z} \partial_{\mathrm{u}} \\
\mathrm{z} \partial_{\mathrm{z}}+(1+b) \mathrm{u} \partial_{\mathrm{u}}\end{array}$ & $\dddot{\mathrm{u}}=C \ddot{\mathrm{u}}^{\frac{b-2}{b-1}}, b \neq 1,2$ & $\begin{aligned} v_{1}:(\mathrm{z}, \mathrm{u}) & \mapsto\left(b_{2}^{2} \mathrm{z}, b_{2}^{1} \mathrm{z}+b_{1}^{1} \mathrm{u}\right) \\
b_{1}^{1} b_{2}^{2} b_{3}^{3} & \neq 0, b_{2}^{2} b_{3}^{3}=b_{1}^{1}\end{aligned}$ \\
\hline$L_{4,4}$ & $\begin{array}{c}\partial_{\mathrm{z}}, \partial_{\mathrm{u}}, \mathrm{z} \partial_{\mathrm{z}}+\mathrm{u} \partial_{\mathrm{u}} \\
\mathrm{u} \partial_{\mathrm{z}}-\mathrm{z} \partial_{\mathrm{u}}\end{array}$ & $\dddot{\mathrm{u}}=\frac{3 \ddot{\mathrm{u}}^{2} \dot{\mathrm{u}}}{1+\dot{\mathrm{u}}^{2}}+C \frac{\ddot{\mathrm{u}}^{2}}{1+\dot{\mathrm{u}}^{2}}$ & $v_{1}:(\mathrm{z}, \mathrm{u}) \mapsto(-\mathrm{z},-\mathrm{u})$ \\
\hline$L_{4,5}^{I}$ & $\partial_{\mathrm{z}}, \partial_{\mathrm{u}}, \mathrm{u} \partial_{\mathrm{u}}, \mathrm{u}^{2} \partial_{\mathrm{u}}$ & $\dddot{\mathrm{u}}=\frac{3}{2} \frac{\ddot{\mathrm{u}}^{2}}{\dot{\mathrm{u}}}+C \dot{\mathrm{u}}$ & $\begin{array}{c}v_{1}:(\mathrm{z}, \mathrm{u}) \mapsto\left(\mathrm{z},-\frac{1}{\mathrm{u}}\right) \\
v_{2}:(\mathrm{z}, \mathrm{u}) \mapsto\left(\mathrm{z}, \frac{1}{\mathrm{u}}\right) \\
v_{3}:(\mathrm{z}, \mathrm{u}) \mapsto\left(-\mathrm{z}, b_{2}^{2} \mathrm{u}\right), b_{2}^{2} \neq 0\end{array}$ \\
\hline$L_{4,5}^{I I}$ & $\begin{array}{c}\partial_{\mathrm{u}}, \mathrm{z} \partial_{\mathrm{z}}+\mathrm{u} \partial_{\mathrm{u}} \\
2 \mathrm{zu} \partial_{z}+\mathrm{u}^{2} \partial_{\mathrm{u}}, \mathrm{z} \partial_{\mathrm{z}}\end{array}$ & $\dddot{\mathrm{u}}=3 \frac{\ddot{\mathrm{u}}^{2}}{\dot{\mathrm{u}}}+C \frac{(2 z \ddot{\mathrm{u}}+\dot{\mathrm{u}})^{\frac{3}{2}}}{\mathrm{z}^{2} \sqrt{\dot{\mathrm{u}}}}$ & $\begin{array}{l}v_{1}:(\mathrm{z}, \mathrm{u}) \mapsto(-\mathrm{z},-\mathrm{u}) \\
v_{2}:(\mathrm{z}, \mathrm{u}) \mapsto\left(c_{1} \mathrm{z}, c_{2} \mathrm{u}\right)\end{array}$ \\
\hline$L_{4,6}$ & $\begin{array}{c}\partial_{\mathrm{u}}, \mathrm{z} \partial_{\mathrm{u}}, \partial_{\mathrm{z}} \\
\mathrm{z} \partial_{\mathrm{z}}+\left(2 \mathrm{u}+\frac{1}{2} \mathrm{z}^{2}\right) \partial_{\mathrm{u}}\end{array}$ & $\dddot{\mathrm{u}}=\operatorname{Cexp}(-\ddot{\mathrm{u}})$ & $\begin{array}{c}v_{1}:(\mathrm{z}, \mathrm{u}) \mapsto\left(b_{3}^{3} \mathrm{z}, b_{3}^{1} \mathrm{z}+b_{1}^{1} \mathrm{u}+\frac{1}{2} b_{3}^{2} b_{3}^{3} \mathrm{z}^{2}\right), \\
b_{1}^{1} b_{2}^{2} b_{3}^{3} \neq 0, b_{2}^{2} b_{3}^{3}=b_{1}^{1} .\end{array}$ \\
\hline
\end{tabular}

Table 5:

\begin{tabular}{|c|c|c|c|}
\hline & Algebra & $\begin{array}{l}\text { Invariant } \\
\text { Equation }\end{array}$ & Discrete Transformations \\
\hline$(a)$ & $\partial_{\mathrm{z}}, \mathrm{z} \partial_{\mathrm{z}}, \mathrm{z}^{2} \partial_{\mathrm{z}}, \partial_{\mathrm{u}}, \mathrm{u} \partial_{\mathrm{u}}, \mathrm{u}^{2} \partial_{\mathrm{u}}$ & $\dddot{\mathrm{u}}=\frac{3}{2} \frac{\mathrm{\ddot { }}^{2}}{\dot{\mathrm{u}}}$ & $\begin{aligned} v_{1}:(\mathrm{z}, \mathrm{u}) & \mapsto(\mathrm{z},-\mathrm{u}) \\
v_{2}:(\mathrm{z}, \mathrm{u}) & \mapsto(-\mathrm{z}, \mathrm{u}) \\
v_{3}:(\mathrm{z}, \mathrm{u}) & \mapsto(-\mathrm{z},-\mathrm{u}) \\
v_{4}:(\mathrm{z}, \mathrm{u}) & \mapsto\left(\frac{1}{\mathrm{z}}, \mathrm{u}\right) \\
v_{5}:(\mathrm{z}, \mathrm{u}) & \mapsto\left(\mathrm{z},-\frac{1}{\mathrm{u}}\right)\end{aligned}$ \\
\hline (b) & $\begin{array}{c}\partial_{z}, \partial_{\mathrm{u}}, \mathrm{z} \partial_{\mathrm{z}}+\mathrm{u} \partial_{\mathrm{u}}, \mathrm{u} \partial_{z}-\mathrm{z} \partial_{\mathrm{u}} \\
\left(\mathrm{z}^{2}-\mathrm{u}^{2}\right) \partial_{\mathrm{z}}+2 \mathrm{zu} \partial_{\mathrm{u}}, 2 \mathrm{zu} \partial_{\mathrm{z}}+\left(\mathrm{u}^{2}-\mathrm{z}^{2}\right) \partial_{\mathrm{u}}\end{array}$ & $\dddot{u}=\frac{3 \ddot{u}^{2} \dot{u}}{1+\dot{u}^{2}}$ & $\begin{array}{c}v_{1}:(\mathrm{z}, \mathrm{u}) \mapsto(\mathrm{z},-\mathrm{u}), \\
v_{2}:(\mathrm{z}, \mathrm{u}) \mapsto(-\mathrm{z}, \mathrm{u}), \\
v_{3}:(\mathrm{z}, \mathrm{u}) \mapsto(-\mathrm{z},-\mathrm{u}), \\
v_{4}:(\mathrm{z}, \mathrm{u}) \mapsto\left(\frac{-\mathrm{z}}{\mathrm{z}^{2}+\mathrm{u}^{2}}, \frac{\mathrm{u}}{\mathrm{z}^{2}+\mathrm{u}^{2}}\right)\end{array}$ \\
\hline
\end{tabular}

Table 6:

\begin{tabular}{|c|c|c|}
\hline Algebra & $\begin{array}{l}\text { Invariant } \\
\text { Equation }\end{array}$ & Discrete Transformations \\
\hline$\partial_{\mathrm{u}}, \mathrm{z} \partial_{\mathrm{u}}, \mathrm{z}^{2} \partial_{\mathrm{u}}, \mathrm{u} \partial_{\mathrm{u}}, \partial_{\mathrm{z}}, 2 \mathrm{z} \partial_{\mathrm{z}}+2 \mathrm{u} \partial_{\mathrm{u}},-\mathrm{z}^{2} \partial_{\mathrm{z}}-2 \mathrm{zu} \partial_{\mathrm{u}}$ & $\dddot{\mathrm{u}}=0$ & $\begin{array}{c}v_{1}:(\mathrm{z}, \mathrm{u}) \mapsto\left(-\frac{1}{\mathrm{z}}, \frac{\mathrm{u}}{\mathrm{z}^{2}}\right) \\
v_{2}:(\mathrm{z}, \mathrm{u}) \mapsto(-\mathrm{z}, \mathrm{z}+\mathrm{u}) \\
v_{3}:(\mathrm{z}, \mathrm{u}) \mapsto\left(\mathrm{z}, \mathrm{z}^{2}-\mathrm{u}\right) \\
v_{4}:(\mathrm{z}, \mathrm{u}) \mapsto(\mathrm{z},-\mathrm{u}) \\
v_{5}:(\mathrm{z}, \mathrm{u}) \mapsto(-\mathrm{z}, \mathrm{u}) \\
v_{6}:(\mathrm{z}, \mathrm{u}) \mapsto(-\mathrm{z},-\mathrm{u})\end{array}$ \\
\hline
\end{tabular}

